\title{
Characterising the 'Txt2Stop' Smoking Cessation Text Messaging Intervention in Terms of Behaviour Change Techniques
}

\author{
Susan Michie, ${ }^{1}$ Caroline Free, ${ }^{2}$ and Robert West ${ }^{3}$ \\ ${ }^{1}$ Department of Clinical, Educational and Health Psychology, University College London, UK \\ ${ }^{2}$ Clinical Trials Research Unit, London School of Hygiene and Tropical Medicine, UK \\ ${ }^{3}$ Health Behaviour Research Centre, Department of Epidemiology and Public Health, University College London, UK
}

\begin{abstract}
The 'Txt2Stop' SMS messaging programme has been found to double smokers' chances of stopping. It is important to characterise the content of this information in terms of specific behaviour change techniques (BCTs) for the purpose of future development. This study aimed to (i) extend a proven system for coding BCTs to text messaging and (ii) characterise Txt2Stop using this system. A taxonomy previously used to specify BCTs in face-to-face behavioural support for smoking cessation was adapted for the Txt2Stop messages and inter-rater reliability for the adapted system assessed. The system was then applied to all the messages in the Txt2Stop programme to determine its profile in terms of BCTs used. The text message taxonomy comprised 34 BCTs. Inter-rater reliability was moderate, reaching a ceiling of $61 \%$ for the core program messages with all discrepancies readily resolved. Of 899 texts delivering BCTs, 218 aimed to maintain motivation to remain abstinent, 870 to enhance self-regulatory capacity or skills, 39 to promote use of adjuvant behaviours such as using stop-smoking medication, 552 to maintain engagement with the intervention and 24 were general communication techniques. The content of Txt2Stop focuses on helping smokers with self-regulation and maintaining engagement with the intervention. The intervention focuses to a lesser extent on boosting motivation to remain abstinent; little attention is given to promoting effective use of adjuvant behaviours such as use of nicotine replacement therapy. As new interventions of this kind are developed it will be possible to compare their effectiveness and relate this to standardised descriptions of their content using this system.
\end{abstract}

Keywords: smoking cessation, digital interventions, text messaging, behaviour change techniques, behavioural intervention, mobile phone

\section{Introduction}

There is a global need for affordable, high-reach interventions to aid smoking cessation (World Health Organisation, 2006). The majority of people in high and lowmiddle income countries have mobile phones and in many of these countries text messaging services are free or nearly free to the user (OFCOM, 2009). A large UK-based trial of an intervention called 'Txt2Stop', based on one that had previously been evaluated in New Zealand (STOMP) (Rodgers et al., 2005) has found a doubling of quit rates at six months (Free et al., 2011). The trial compared Txt2Stop with an intervention containing text messages unrelated to quitting in 5,800 smokers and analysed their effect on cessation by intention to treat. Biochemically verified continuous abstinence at six months was $10.7 \%$ in Txt2Stop versus $4.9 \%$ in the control, a rate ratio of 2.10 (95\% CI 1.80 to 2.68). This opens the way for similar types of intervention to be developed in other countries. In order to be able to do this it is essential that the nature and content of the intervention is described using a system that can be extrapolated beyond the current version.

Recent research in behaviour change has identified reliable methods for specifying the content of interventions in terms of component behaviour change techniques 
(BCTs) (Michie, Abraham et al., 2011). Taxonomies of BCTs have been developed and applied in relation to interventions to aid smoking cessation, reduce excessive alcohol consumption (Michie, Whittington, Hamoudi, Zarnani, Tober \& West, 2012), improve diet, increase physical activity and prevent recurrent cardiovascular disease (Michie, Ashford, et al., 2011; Michie, Hyder, Walia \& West, 2011; West, Evans \& Michie, 2011; Redfern et al., 2012). This approach has allowed the identification of 'active ingredients' within multi-component interventions in these domains (West, Walia, Hyder, Shahab \& Michie, 2010; Michie, Abraham, Whittington, McAteer \& Gupta, 2009). Studies detecting associations between inclusion of certain BCTs in behavioural interventions and effectiveness have been conducted both in relation to national datasets of validated one month quit rates (West et al., 2010) and in relation to systematic reviews (Michie et al., 2009). BCTs for behavioural support in smoking cessation have been grouped according to their functions in bringing about behaviour change, i.e. linked to mechanisms of change (Michie, Churchill \& West, 2011).

Although smokers using Txt2Stop were twice as likely to be continuously abstinent at six months as those who were not, $90 \%$ were smoking at this point. Therefore, there is scope to improve this intervention. A first step is to understand how the intervention works, in other words, the "active ingredients" within the intervention. To achieve this, it is necessary to identify the specific BCTs within the intervention. This paper describes the development of a BCT taxonomy for specifying the content of smoking cessation interventions and characterizes Txt2Stop in terms of its component BCTs.

Txt2Stop consists of 186 core behaviour change text messages combined with additional personalised messages selected from a database of 713 messages. These use an algorithm based on demographic and personal interests, for example smokers' concerns about weight gain following quitting or interest in receiving text messages about the unethical tactics of the tobacco companies. Participants are asked to set a quit date within 14 days of randomization, and receive five text messages per day in the first five weeks and a further three per week for six months. Participants can opt to have a 'quit buddy' where participants with similar characteristics and quit dates are paired up so that they can text each other for support. If they want help to deal with craving, they can also text the word 'crave' and receive an immediate text message randomly selected from a database of 128 messages. If participants smoke they can text the word 'lapse' and a series of three messages are delivered in response from a database of 47 messages.

Txt2Stop was adapted from the STOMP text messages. As a result of user testing amongst 62 smokers, ex-smokers and smokers trying to quit, messages were discarded, modified or added (for more details, see Free, 2012; Free, Whittaker, Knight, Abramsky, Rodgers \& Roberts, 2009).
This study aimed to (i) extend the BCT coding method to characterise text-messaging interventions and (ii) use the method to specify the content of 'Txt2Stop', the first text messaging smoking cessation intervention with clearly established efficacy.

\section{Methods \\ Development of the coding method}

Two coders independently analysed the 186 core behaviour change messages using a taxonomy of $43 \mathrm{BCTs}$ previously found to be reliable in coding the context of treatment manuals for face-to-face behavioural support for smoking cessation (Michie, Hyder, et al., 2011). The coding instructions were developed from this coding scheme by the coders. Coders were health psychology researchers with a background in behaviour change interventions and knowledge of smoking cessation. Coding ambiguities arose when the text messages were brief, did not have a very clear behaviour change target or there was more than one target, with one message involving more than one BCT. This led to a revision of the coding instructions such that if a text message clearly included more than one BCT, both would be identified, and if a text message did not relate clearly to a specific BCT it would be labelled as 'not codable'. An example of the former is "Making change is hard but the reward is huge. More money, better health, easier life. Keep thinking of yourself as a non-smoker", which comprises the BCTs 'Provide information on consequences of smoking and smoking cessation' and 'Strengthen ex-smoker identity'. There were also 128 'crave' texts and 47 'lapse' texts and 538 'personal interest' texts, which comprised information selected on the basis of participants' demographic and personal interests

In addition, it was found that two BCTs ('facilitate restructuring of social life', 'advise on avoidance of social cues for smoking') that were differentiable in face-to-face support could not easily be differentiated in text messages and these were merged. Moreover, five BCTs were identified that had not been part of face-to-face support: 'promote behavioural substitution' 'provide behavioural substitution', 'promote engagement with programme', 'promote self-reward', and 'create or reinforce negative associations'. The second of these arose from the fact that the text message could act in real time as a substitute for smoking. All 'crave' text messages were coded as providing a real time substitute for smoking. The third BCT arose from the fact that maintaining contact with the programme on a daily basis was an important part of the intervention. All 538 personal interest text messages were coded as promoting engagement with the program and providing behavioural substitution. The fourth and fifth new BCTs emerged because the modality of communication made it feasible to present brief communications to foster negative emotional associations with smoking and to prompt 
the user to provide rewards for achievements as the course progressed.

\section{Classification of text messages by BCT}

Once the coding system had been finalised, all 899 Txt2Stop messages were coded to develop a profile of BCTs classified according to their function. The texts were independently coded by CF and SM, and discrepancies were discussed and agreed by RW and SM. The content of the messages was then characterised as a profile of the number of different types of BCT used and the number of BCTs classified according to their function. It should be noted that one text could have more than one BCT.

\section{Results}

\section{The coding method}

Sixty-one per cent (113) of the 186 core programme text messages were classified exactly the same by independent coders using the revised coding system. This included multiple BCTs within many of the messages. Although the level of agreement was relatively low compared with previous BCT coding schemes, differences arose from subtly different interpretations of the intent behind some of the text messages and these were readily resolved through discussion. Minor changes were made to BCT wording and coding instructions (see Web Supplement for final coding guidelines). Of $47 \mathrm{BCTs}$ that were potentially incorporated into Txt2Stop ( 43 from the face-to-face interventions, two merged and five added), only 34 were eventually used. Others were either not appropriate (e.g. measure expiredair $\mathrm{CO}$ ), were judged by the intervention designers not to be required (e.g. set graded tasks) or appropriate (e.g. provide feedback on current behaviour), or may not have been considered when designing the intervention (e.g. prompt self-recording).

\section{Classification of text messages by BCT}

The frequency of BCTs in each of the types of Txt2Stop message is shown in Table 1.

They are grouped according to their function in bringing about behaviour change. Of the 1,703 instances of BCTs in the Txt2Stop intervention, 218 were aimed at maintaining motivation to remain abstinent, 870 at enhancing self-regulatory capacity or skills, 39 at promoting use of adjuvant behaviours such as using stop-smoking medication (NRT), 552 at maintaining engagement with the intervention and 24 were general communication techniques. Thus, all intervention functions were represented by BCTs.

Over $90 \%$ of the instances of BCTs involved just seven BCTs. Three were motivational: 'provide information on consequences and consequences', 'boost motivation and self-efficacy', 'create or reinforce negative associations'; three were self-regulatory: 'promote use of relaxation techniques', 'promote behavioural substitution' and 'provide behavioural substitution'; and one was related to intervention delivery: 'promote engagement with the programme'.

\section{Discussion}

The results demonstrate the possibility of developing a method to characterize an effective smoking cessation text-messaging intervention in terms of its component BCTs.

In previous research, taxonomies of BCTs have been used to characterise interventions as described in intervention protocols or published reports, with good reliability. The reliability of using this method to characterise text messages was lower. The coders, who were experienced in coding both types of material, have provided several possible explanations for this. Text messages are extremely brief, stand-alone messages, without context, providing less to draw on in judging the intent of the words. In addition, the telescoped language gives rise to ambiguity of interpretation and each message may contain one, more or no BCTs. It should be noted that Txt2Stop was designed before the availability of a taxonomy of BCTs. If future intervention designers use a taxonomy of BCTs to design their interventions, the specification will be more precise and there will not be the need for post-hoc coding with its associated problems of inter-rater reliability.

Thirty-four BCTs were identified, although two-thirds of the texts contained one or more of just six BCTs. These served to boost motivation and promote self-regulatory activities. To our knowledge, this is first time that an attempt has been made systematically to specify a text messaging smoking cessation intervention in terms of its component BCTs. It provides a basis for experimental manipulation of intervention content to ascertain which components are the active ingredients in the intervention, paving the way for designing more effective interventions.

Future text messaging interventions may benefit from more frequent use of the BCTs identified in this research, such as 'strengthen ex-smoker identity', 'provide reassurance' and 'advise on use of social support'. Txt2Stop included one BCT that had not been identified in smoking cessation guidelines, protocols or published reports (Michie, Churchill, et al., 2011) but had been previously identified in interventions to reduce excessive alcohol literature (Michie, Whittington, et al., 2012): 'Behavioural substitution'. This was used in three ways: suggestions for substituting activities were given, texting from the mobile phone was itself used as a substitution for participants using the text 'crave' function, and receiving personal interest messages was used as substitution.

Taxonomies of BCTs continue to be developed (Michie, Johnston, et al., 2012; Michie, Whittington, et al., 2012) allowing for more accurate characterisation of interventions (see www.ucl.ac.uk/health-psychology/BCTtaxonomy/). For example, some messages provided feedback to participants about the health and physical benefits they had achieved by quitting. The message 'Health update! Well 
TABLE 1

Frequency of behaviour change techniques, grouped according to their function, and tabulated by type of Txt2Stop message (codes in the first column are based on those in Michie, Churchill \& West, 2011)

\begin{tabular}{|c|c|c|c|c|c|c|}
\hline BCT code and function & BCT label & $\begin{array}{l}\text { Behaviour change } \\
\text { texts }(n=186)\end{array}$ & $\begin{array}{l}\text { Crave texts } \\
(\mathrm{n}=128)\end{array}$ & $\begin{array}{l}\text { Lapse texts } \\
(\mathrm{n}=47)\end{array}$ & $\begin{array}{l}\text { Personal interest } \\
\text { texts }(n=538)\end{array}$ & $\begin{array}{l}\text { TOTAL } \\
(\mathrm{n}=899)\end{array}$ \\
\hline BM1 & $\begin{array}{l}\text { Provide information on } \\
\text { consequences of smoking } \\
\text { and smoking cessation }\end{array}$ & 20 & 9 & 1 & 12 & 42 \\
\hline BM2 & $\begin{array}{l}\text { Boost motivation and } \\
\text { self-efficacy }\end{array}$ & 21 & 18 & 27 & 45 & 111 \\
\hline BM4 & $\begin{array}{l}\text { Provide rewards contingent } \\
\text { on successfully stopping } \\
\text { smoking }\end{array}$ & 10 & 1 & 1 & & 12 \\
\hline BM5 & $\begin{array}{l}\text { Provide normative } \\
\text { information about others' } \\
\text { behaviour and experiences }\end{array}$ & 2 & & & & 2 \\
\hline BM6 & $\begin{array}{l}\text { Prompt commitment from the } \\
\text { client there and then }\end{array}$ & & & & 1 & 1 \\
\hline BM8 & $\begin{array}{l}\text { Strengthen ex-smoker } \\
\text { identity }\end{array}$ & 8 & & & & 8 \\
\hline BM10 & $\begin{array}{l}\text { Identify reasons for wanting } \\
\text { and not wanting to stop } \\
\text { smoking }\end{array}$ & 5 & & & & 5 \\
\hline BM11 & $\begin{array}{l}\text { Explain the importance of } \\
\text { abrupt cessation }\end{array}$ & & & & 1 & 1 \\
\hline BM13 & $\begin{array}{l}\text { Create or reinforce negative } \\
\text { associations }\end{array}$ & 7 & 3 & & 25 & 35 \\
\hline BM14 & $\begin{array}{l}\text { Distract from motivation to } \\
\text { engage in behaviour }\end{array}$ & & & & 2 & 2 \\
\hline Total motivational & & 72 & 31 & 29 & 86 & 218 \\
\hline BS1 & $\begin{array}{l}\text { Facilitate barrier } \\
\text { identification and problem } \\
\text { solving }\end{array}$ & 6 & & & & 6 \\
\hline BS2 & $\begin{array}{l}\text { Facilitate relapse prevention } \\
\text { and coping }\end{array}$ & 8 & & 7 & & 15 \\
\hline BS3 & $\begin{array}{l}\text { Facilitate action } \\
\text { planning/develop } \\
\text { treatment plan }\end{array}$ & 4 & & 1 & 1 & 6 \\
\hline BS4 & Facilitate goal setting & 1 & & & & 1 \\
\hline BS5 & Prompt review of goals & 2 & & & & 2 \\
\hline BS7 & Advise on changing routine & 6 & 2 & & & 8 \\
\hline BS8 & $\begin{array}{l}\text { Advise on environmental } \\
\text { restructuring }\end{array}$ & 7 & 1 & & 1 & 9 \\
\hline BS9 & Set graded tasks & 7 & & & & 7 \\
\hline BS10 & $\begin{array}{l}\text { Advise on conserving mental } \\
\text { resources }\end{array}$ & 3 & 4 & & & 7 \\
\hline BS11 & $\begin{array}{l}\text { Advise on avoidance of cues } \\
\text { for smoking }\end{array}$ & 3 & 2 & & 3 & 8 \\
\hline BS12 & $\begin{array}{l}\text { Facilitate restructuring of } \\
\text { social life }\end{array}$ & 2 & & & 1 & 3 \\
\hline BS13 & $\begin{array}{l}\text { Advise on methods of weight } \\
\text { control }\end{array}$ & 7 & & & & 7 \\
\hline BS14 & $\begin{array}{l}\text { Promote use of relaxation } \\
\text { techniques }\end{array}$ & 2 & & & 78 & 80 \\
\hline
\end{tabular}


TABLE 1

(Continued)

\begin{tabular}{|c|c|c|c|c|c|c|}
\hline $\mathrm{BCT}$ code and function & BCT label & $\begin{array}{l}\text { Behaviour change } \\
\text { texts }(n=186)\end{array}$ & $\begin{array}{l}\text { Crave texts } \\
(\mathrm{n}=128)\end{array}$ & $\begin{array}{l}\text { Lapse texts } \\
(\mathrm{n}=47)\end{array}$ & $\begin{array}{l}\text { Personal interest } \\
\text { texts }(n=538)\end{array}$ & $\begin{array}{l}\text { TOTAL } \\
(\mathrm{n}=899)\end{array}$ \\
\hline BS15 & $\begin{array}{l}\text { Promote behavioural } \\
\text { substitution }\end{array}$ & 28 & 13 & 8 & 49 & 98 \\
\hline BS16 & Promote self-reward & 7 & & & & 7 \\
\hline BS17 & $\begin{array}{l}\text { Provide behavioural } \\
\text { substitution }\end{array}$ & & 60 & & 538 & 666 \\
\hline Total self-regulation & & 103 & 80 & 16 & 671 & 870 \\
\hline $\mathrm{A} 1$ & $\begin{array}{l}\text { Advise on stop-smoking } \\
\text { medication }\end{array}$ & 7 & 1 & 1 & & 9 \\
\hline$A 2$ & $\begin{array}{l}\text { Advise on/facilitate use of } \\
\text { social support }\end{array}$ & 11 & 6 & 1 & 8 & 26 \\
\hline A5 & $\begin{array}{l}\text { Give options for additional } \\
\text { and later support }\end{array}$ & 4 & & & & 4 \\
\hline Total adjunctive & & 22 & 7 & 2 & 8 & 39 \\
\hline RD3 & $\begin{array}{l}\text { Promote engagement with } \\
\text { the programme }\end{array}$ & 14 & & & 538 & 552 \\
\hline Total intervention delivery & & 14 & 0 & 0 & 538 & 552 \\
\hline $\mathrm{RC2}$ & Elicit and answer questions & 2 & & & & 2 \\
\hline RC4 & $\begin{array}{l}\text { Explain expectations } \\
\text { regarding treatment } \\
\text { programme }\end{array}$ & 8 & & & & 8 \\
\hline $\mathrm{RC} 6$ & $\begin{array}{l}\text { Provide information on } \\
\text { withdrawal symptoms }\end{array}$ & 5 & & & & 5 \\
\hline RC10 & Provide reassurance & 9 & & & & 9 \\
\hline Total general communication & & 24 & 0 & 0 & 0 & 24 \\
\hline TOTAL number of BCTs & & 235 & 118 & 47 & 1303 & 1703 \\
\hline
\end{tabular}

done, your oxygen levels are now normal' was specifically timed to be sent on the quit day to arrive when oxygen levels would be normal. Since this is not 'feedback on current behaviour', this type of message was coded as 'provide information on the consequences of the behaviour'. In the most recent, integrated taxonomy, there is a new, more specific BCT, 'Health consequences', defined as 'Record/provide information about health consequences of performing the behaviour'.

Reliable coding of interventions requires close attention to the definition of each BCT and a good knowledge as to how it differs from others. It is a skill that requires both training and practice. Good results have been achieved from intensive one-day workshops, and long-distance tutorial training is currently being evaluated. A further development that may be helpful to intervention designers and reporters is the establishment of a hierarchical structure, with 16 groupings of BCTs identified (Michie, Johnston, et al., 2012). The aim of this structure is to make the taxonomy more conceptually coherent and easier to use.

The impact of the Txt2Stop intervention on specific smoking cessation attitudes and behaviours has been evaluated in a subsample of 600 participants. Qualitative in- terviews with participants about their experiences of the intervention provided further insight into the types of messages that participants found most and least helpful and some ideas about the possible mechanisms of action of the intervention. This will be reported elsewhere.

Future text messaging interventions are likely to benefit from drawing on both the method developed in this study and the more generic work investigating efficacy of the component BCTs. The data from the current study, together with BCTs found to be effective in other modalities but not currently found in Txt2Stop, would constitute useful information to underpin the next version of a textmessaging intervention. This approach should also be extended to other low-cost, high-reach distance behavioural support, such as telephone support and internet-based support (Michie, Brown et al., 2012; Brown, Michie et al., 2012).

\section{Funding}

The authors thank the Medical Research Council for funding the Txt2Stop trial. Robert West is funded by Cancer Research UK. 


\section{Declaration of interests}

Robert West undertakes research and consultancy for companies that manufacture smoking cessation medications. He is also a trustee of QUIT and a member of the UK Centre for Tobacco Control Studies.

\section{Acknowledgments}

We are grateful to Felix Naughton, Maartje van Stralen, Eline de Smit, Eleni Vangeli, Lion Shahab, Fabi Lorencatto and Natalie Seymour for their work in coding the Txt2Stop messages.

\section{References}

Brown, J., Michie, S., Geraghty, A. W., Miller, S., Yardley, L., Gardner, B., Shahab, L., Stapleton, J. A., \& West, R. (2012). A pilot study of StopAdvisor: a theory-based interactive internet-based smoking cessation intervention aimed across the social spectrum. Addictive Behaviors. doi: 10.1016/j.addbeh.2012.05.016

Free, C. (2012). Developing and adapting a text messaging intervention for smoking cessation from New Zealand for the United Kingdom. In J. Donner and P. Mechael (Eds.), Healthy Behaviors: Research and practice in the use of mobile technology for health promotion. London: Bloomsbury.

Free, C., Whittaker, R., Knight, R., Abramsky, T., Rodgers, A., \& Roberts, I. G. (2009). Txt2Stop: a pilot randomised controlled trial of mobile phone-based smoking cessation support. Tobacco Control, 18(2), 88-91. doi: $10.1136 /$ tc. 2008.026146

Free, C., Knight, R., Robertson, S., Whittaker, R., Edwards, P., Zhou, W., et al. (2011). Smoking cessation support delivered via mobile phone text messaging (Txt2Stop): A single-blind, randomised trial. Lancet, 378, 48-55. doi: 10.1016/S01406736(11)60701-0

Michie, S., Abraham, C., Eccles, M. P., Francis, J. J., Hardeman, W., \& Johnston, M. (2011). Strengthening evaluation and implementation by specifying components of behaviour change interventions: a study protocol. Implementation Science, 6(1), 10. doi: 10.1186/1748-5908-6-10

Michie, S., Abraham, C., Whittington, C., McAteer, J., \& Gupta, S. (2009). Effective techniques in healthy eating and physical activity interventions: a meta-regression. Health Psychology, 28(6), 690-701. doi: 10.1037/a0016136

Michie, S., Ashford, S., Sniehotta, F. F., Dombrowski, S. U., Bishop, A., \& French, D. P. (2011). A refined taxonomy of behaviour change techniques to help people change their physical activity and healthy eating behaviours: The CALORE taxonomy. Psychology \& Health, 26, 1479-1498.

Michie, S., Brown, J., Geraghty, A., Miller, S., Yardley, L., Gardner, B., Shahab, L., McEwen, A., Stapleton, J., \& West, R. (2012).
Development of StopAdvisor, a theory-based interactive, internet-based smoking cessation intervention. Translational Behavioral Medicine. doi: 10.1007/s13142-012-0135-6

Michie, S., Churchill, S., \& West, R. (2011). Identifying evidencebased competencies required to deliver behavioural support for smoking cessation. Annals of Behavioural Medicine, 41, 59-70. doi: 10.1007/s12160-010-9235-Z

Michie, S., Hyder, N., Walia, A., \& West, R. (2011). Development of a taxonomy of behaviour change techniques used in individual behavioural support for smoking cessation. Addictive Behaviours, 36(4), 315-319. doi: 10.1016/j.addbeh.2010.11.016

Michie, S., Johnston, M., Abraham, C., Francis, J., Hardeman, W., Eccles, M., et al. (2012). An 87-item taxonomy of behavior change techniques: Building an international consensus for the reporting of behavior change interventions. Paper presented at 33rd Annual Meeting of the Society of Behavioral Medicine, New Orleans, USA, April 2012.

Michie, S., Whittington, C., Hamoudi, Z., Zarnani, F., Tober, G. \& West, R. (2012). Behaviour change techniques to reduce excessive alcohol use and their associations with outcome.

OFCOM (2009). The Consumer Experience: Telecoms, Internet and Digital Broadcasting. Evaluation Report. London. Retrieved from: http://stakeholders.ofcom.org.uk/marketdata-research/market-data/consumer-experience-reports/ eval09/.

Redfern, R., Thiagalingam, A., Jan, S, Whittaker, R., Hackett, M.L., Mooney, J., De Keizer, L., Hillis, G.S., \& Chow, C.S. (2012). Development of a set of mobile phone text messages designed for prevention of recurrent cardiovascular events. European Journal of Preventive Cardiology, published online 17 May 2012.

Rodgers, A., Corbett, T., Bramley, D., Riddell, T., Wills, M., Lin, R. B., et al. (2005). Do u smoke after txt? Results of a randomised trial of smoking cessation using mobile phone text messaging. Tobacco Control, 14(4), 255-261. doi: 10.1136/tc.2005.011577

West, R., Walia, A., Hyder, N., Shahab, L., \& Michie, S. (2010). Behaviour change techniques used by the English Stop Smoking Services and their associations with short-term quit outcomes. Nicotine and Tobacco Research, 12, 742-747. doi:10.1093/ntr/ntq074

West, R., Evans, A., \& Michie, S. (2011). Behaviour change techniques used in group-based behavioural support by the English Stop-Smoking Services and preliminary assessment of association with short-term outcomes. Nicotine and Tobacco Research, 13(12):1316-1320.

World Health Organisation (2006). WHO Framework Convention on Tobacco Control. Geneva: World Health Organisation. 